Bull. Austral. Math. Soc.

VOL. 42 (1990) [287-292]

\title{
TRANSLATION INVARIANT LINEAR FUNCTIONALS ON SEGAL ALGEBRAS
}

\section{YUJI TAKAHASHI}

Let $S(G)$ be a Segal algebra on an infinite compact Abelian group $G$. We study the existence of many discontinuous translation invariant linear functionals on $S(G)$. It is shown that if $G / C_{G}$ contains no finitely generated dense subgroups, then the dimension of the linear space of all translation invariant linear functionals on $S(G)$ is greater than or equal to $2^{c}$ and there exist $2^{c}$ discontinuous translation invariant linear functionals on $S(G)$, where $c$ and $C_{G}$ denote the cardinal number of the continuum and the connected component of the identity in $G$, respectively.

Throughout this note $G$ will denote an infinite compact Abelian group with the normalised Haar measure $\lambda_{G}$, and $L^{p}(G)(1 \leqslant p \leqslant \infty)$ will denote the Lebesgue space with respect to $\lambda_{G}$. The space of all continuous functions on $G$ will be denoted by $C(G)$. We shall also use the symbols $c$ and $C_{G}$ to denote the cardinal number of the continuum and the connected component of the identity in $G$, respectively.

Roelcke, Asam, S.Dierolf and P. Dierolf [9, Theorem 4] proved that if $G$ is a torsion group, then the dimension of the linear space of all translation invariant linear functionals on $C(G)$ is greater than or equal to $2^{c}$. This result in particular implies that $C(G)$ admits $2^{c}$ discontinuous translation invariant linear functionals for any infinite compact Abelian torsion group $G$. The existence of discontinuous translation invariant linear functionals on $L^{2}(G)$ was studied by Meisters [6]. Recall that a compact Abelian group is called polythetic if it contains a finitely generated dense subgroup (see $[2,6])$. Meisters, together with Larry Baggett, proved that $L^{2}(G)$ has discontinuous translation invariant linear functionals provided that $G / C_{G}$ is not polythetic [6, Corollary to Theorem 6]. The purpose of this note is to indicate how the methods in [9] may be improved to establish a theorem which strengthens and generalises the above two results.

For a function $f$ on $G$ and $a \in G$, we define the $a$-translate $r(a) f$ of $f$ by $(\tau(a) f)(x)=f(x-a)(x \in G)$. Recall that, by definition, a Segal algebra on $G$ is a dense subalgebra $S(G)$ of the convolution algebra $L^{1}(G)$ such that

(i) $S(G)$ is a Banach algebra under some norm $\|\cdot\|_{S}$ and $\|f\|_{S} \geqslant\|f\|_{L^{1}}$ for all $f \in S(G)$;

Received 2 November 1989

Copyright Clearance Centre, Inc. Serial-fee code: 0004-9729/90 \$A2.00+0.00. 
(ii) $S(G)$ is translation invariant (that is, $\tau(a) f \in S(G)$ for all $f \in S(G)$ and all $a \in G)$ and for each $f \in S(G)$ the mapping $a \rightarrow \tau(a) f$ of $G$ into $S(G)$ is continuous;

(iii) $\|\tau(a) f\|_{S}=\|f\|_{S}$ for all $f \in S(G)$ and all $a \in G$.

(For fundamental results on Segal algebras, we refer to $[7,8,12]$.) We say that a linear functional $\Phi$ on a Segal algebra $S(G)$ is translation invariant if $\Phi(\tau(a) f)=\Phi(f)$ for all $f \in S(G)$ and all $a \in G$. In this note we shall be concerned with translation invariant linear functionals on Segal algebras on $G$. Henceforth we shall use the abbreviation TILF for "translation invariant linear functional" and denote by TILF $(S(G))$ the linear space of all TILF's on $S(G)$.

Let us now state our theorem.

TheOREM. Let $G$ be a compact Abelian group and let $S(G)$ be a Segal algebra on $G$. If $G / C_{G}$ is not polythetic, then the dimension of the linear space $\operatorname{TILF}(S(G))$ is greater than or equal to $2^{c}$ and there exist $2^{c}$ discontinuous TILF's on $S(G)$.

To prove our Theorem, we require some preliminary notation and lemmas. $\widehat{G}$ will denote the (discrete) dual group of a compact Abelian group. (We use $1_{G}$ to denote the trivial character of $G$.) For $f \in L^{1}(G), \hat{f}$ denotes the Fourier transform of $f$. For a Segal algebra $S(G)$, we denote by $\Delta(S(G))$ and $S(G)_{0}$ the linear subspace of $S(G)$ generated by $\{f-\tau(a) f: f \in S(G), a \in G\}$ and the closed linear subspace $\left\{f \in S(G): \widehat{f}\left(1_{G}\right)=0\right\}$ of $S(G)$, respectively. Then it is clear that $S(G)_{0}$ contains $\triangle(S(G))$.

Lemma 1. Let $G$ be a compact Abelian group and let $S(G)$ be a Segal algebra on $G$. Then the closure $\overline{\triangle(S(G))}$ in $S(G)$ equals $S(G)_{0}$ and every continuous TILF on $S(G)$ is a scalar multiple of the Haar integral.

Proof: For a subset $E$ of $L^{1}(G)$, we denote by $\bar{E}^{L^{1}}$ the closure of $E$ in the $L^{1}$-norm. Since ${\overline{\Delta\left(L^{1}(G)\right)}}^{L^{1}}=L^{1}(G)_{0}\left(\left[4\right.\right.$, Lemma 1.1]) and $S(G)$ is dense in $L^{1}(G)$, we have

$$
\overline{\Delta(S(G))}^{L^{1}}={\overline{\Delta\left(L^{1}(G)\right)}}^{L^{1}}=L^{1}(G)_{0} .
$$

Notice that $\overline{\triangle(S(G))}$ is a closed ideal of $S(G)$. Thus it follows from [12, Theroem 4.3] that

$$
\overline{\Delta(S(G))}=\overline{\overline{\Delta(S(G))}}{ }^{1} \cap S(G) \text {. }
$$

Hence we have

$$
\begin{aligned}
S(G)_{0} & =L^{1}(G)_{0} \cap S(G)=\overline{\Delta(S(G))}^{L^{1}} \cap S(G) \\
& \cong \overline{\overline{\Delta(S(G))}}^{{ }^{1}} \cap S(G)=\overline{\Delta(S(G))} .
\end{aligned}
$$


Since the converse inclusion relation is clear, we conclude that $\overline{\Delta(S(G))}=S(G)_{0}$. Let $\Phi$ be a continuous TILF on S(G). Then, of course, $\Phi \equiv 0$ on $\Delta(S(G))$ and hence on $\overline{\Delta(S(G))}$. Since $\overline{\Delta(S(G))}=S(G)_{0}$ and $S(G)_{0}$ has codimension one, either $\Phi$ is identically zero or the kernel of $\Phi$ coincides with $S(G)_{0}$. In either case $\Phi$ is a scalar multiple of the Haar integral. This completes the proof.

LEMma 2. Let $G$ be an infinite metrisable compact $A$ belian group and let $S(G)$ be a Segal algebra. Then there exists a family $\left\{h_{r}\right\}_{r>1}$ (indexed by real numbers $r$ with $r>1$ ) of functions in $S(G)$ with the following properties:

(i) $\widehat{h}_{r}\left(1_{G}\right)=0$ for every $r>1$,

(ii) $\{\gamma \in \widehat{G}: \widehat{h}(\gamma)=0\}$ is finite for every nonzero function $h$ in the linear space generated by $\left\{h_{r}\right\}_{r>1}$.

Proof: Since $\widehat{G}$ is countably infinite, we denote $\widehat{G}$ by $\left\{\gamma_{0}=1_{G}, \gamma_{1}, \gamma_{2}, \ldots\right.$, $\left.\gamma_{n}, \ldots\right\}$. For each $r>1$, we define a function $h_{r}$ in $S(G)$ by

$$
h_{r}=\sum_{n=1}^{\infty} n^{-r}\left\|\gamma_{n}\right\|_{S}^{-1} \gamma_{n} .
$$

See, for example, Theorem 4.2 of [12]. (Note that the series of the right side converges in $S(G)$.) It is easy to see that $\widehat{h}_{r}\left(1_{G}\right)=0$ and $\widehat{h}_{r}\left(\gamma_{n}\right)=n^{-r}\left\|\gamma_{n}\right\|_{S}^{-1}$ for all $n \geqslant 1$. Thus (i) holds. To see (ii), let $h=\sum_{j=1}^{m} c_{j} h_{r_{j}}$ be a nonzero function in the linear space generated by $\left\{h_{r}\right\}_{r>1}$, where $c_{j}(1 \leqslant j \leqslant m)$ is a nonzero complex number and $1<r_{1}<r_{2}<\ldots<r_{m}$. Since

$$
\begin{aligned}
\left|\widehat{h}\left(\gamma_{n}\right)\right| & =\left|\sum_{j=1}^{m} c_{j} \widehat{h}_{r_{j}}\left(\gamma_{n}\right)\right| \\
& =\left|\sum_{j=1}^{m} c_{j} n^{-r_{j}}\left\|\gamma_{n}\right\|_{S}^{-1}\right| \\
& =n^{-r_{1}}\left\|\gamma_{n}\right\|_{S}^{-1}\left|\sum_{j=1}^{m} c_{j} n^{r_{1}-r_{j}}\right| \\
& \geqslant n^{-r_{1}}\left\|\gamma_{n}\right\|_{S}^{-1}\left(\left|c_{1}\right|-\sum_{j=2}^{m}\left|c_{j}\right| n^{r_{1}-r_{j}}\right)
\end{aligned}
$$

for all $n \geqslant 1$, we have $\hat{h}\left(\gamma_{n}\right) \neq 0$ for all sufficiently large positive integers $n$ and hence (ii) holds. This completes the proof. 
Let us now turn to the proof of the Theorem. We shall show that the dimension of the linear space $S(G)_{0} / \Delta(S(G))$ is greater than or equal to $c$. This immediately implies that

$$
\operatorname{dim} \operatorname{TILF}(S(G)) \geqslant 2^{c} .
$$

Since the linear space of all continuous TILF's on $S(G)$ has dimension one by Lemma 1 , we also obtain that there exist $2^{c}$ discontinuous TILF's on $S(G)$.

We first consider the case where $G$ is metrisable and not polythetic. Let $\left\{h_{r}\right\}_{r>1}$ be a family of functions in $S(G)$ as in Lemma 2 and let $X$ denote the linear subspace of $S(G)$ generated by $\left\{h_{r}\right\}_{r>1}$. Then, by Lemma $2(i), X$ is included in $S(G)_{0}$. We also have

$$
X \cap \triangle(S(G))=\{0\} .
$$

To see this, suppose that there exist $f_{1}, f_{2}, \ldots, f_{n} \in S(G)$ and $a_{1}, a_{2}, \ldots, a_{n} \in G$ such that

$$
f=\sum_{j=1}^{n}\left(f_{j}-\tau\left(a_{j}\right) f_{j}\right)
$$

is nonzero and is contained in $X$. Then, by Lemma 2 (ii), there exist only finitely many $\gamma_{1}, \gamma_{2}, \ldots, \gamma_{m} \in \widehat{G} \backslash\left\{1_{G}\right\}$ such that $\widehat{f}\left(\gamma_{k}\right)=0$ for $k=1,2, \ldots, m$. Choose $b_{1}, b_{2}, \ldots, b_{m} \in G$ such that $\gamma_{k}\left(b_{k}\right) \neq 1$ for $k=1,2, \ldots, m$ and denote by $H$ the closed subgroup of $G$ generated by $\left\{a_{1}, \ldots, a_{n}, b_{1}, \ldots, b_{m}\right\}$. (If $\{\gamma \in \widehat{G}: \widehat{f}(\gamma)=0\}=$ $\left\{1_{G}\right\}$, then we simply consider the closed subgroup $H$ of $G$ generated by $\left\{a_{1}, \ldots, a_{n}\right\}$.) Since $G$ is not polythetic, $H$ is proper in $G$ and hence there exists $\gamma \in \widehat{G} \backslash\left\{1_{G}\right\}$ such that $\gamma(x)=1$ for all $x \in H$. Then we have

$$
0 \neq \hat{f}(\gamma)=\sum_{j=1}^{n}\left(1-\overline{\gamma\left(a_{j}\right)}\right) \hat{f}_{j}(\gamma)=0 .
$$

But this is a contradiction, and hence $X \cap \Delta(S(G))=\{0\}$ as desired. Thus we obtain

$$
\operatorname{dim} S(G)_{0} / \triangle(S(G)) \geqslant \operatorname{dim} X .
$$

Since $\operatorname{dim} X=c$ by Lemma 2 (ii), we conclude that

$$
\operatorname{dim} S(G)_{0} / \Delta(S(G)) \geqslant c
$$

We next turn to the general case. Since $G / C_{G}$ is not polythetic, there exists a closed subgroup $H$ of $G$ such that $G / H$ is metrisable and not polythetic ([2], Lemma 5.2). Notice that we can define a bounded linear operator $T_{H}$ from $L^{1}(G)$ onto $L^{1}(G / H)$ as follows:

$$
T_{H}(f)(x+H)=\int_{H} f(x+\xi) d \lambda_{H}(\xi) \quad\left(f \in L^{1}(G), x \in G\right) .
$$


By [8, Section 13, Theorem 1], the image of $S(G)$ under $T_{H}$ is a Segal algebra on $G / H$. Let us denote by $S(G / H)$ this Segal algebra. Then it can be easily verified that the image of $S(G)_{0}$ under $T_{H}$ coincides with $S(G / H)_{0}$ and that $\Delta(S(G))=T_{H}^{-1}(\Delta(S(G / H)))$. Thus $S(G)_{0} / \triangle(S(G))$ is linearly isomorphic with $S(G / H)_{0} / \Delta(S(G / H))$. Since $G / H$ is metrisable and not polythetic, we have

$$
\operatorname{dim} S(G)_{0} / \Delta(S(G))=\operatorname{dim} S(G / H)_{0} / \Delta(S(G / H)) \geqslant c .
$$

This completes the proof of the Theorem.

REMARKS. (a) If $G$ is an infinite compact Abelian torsion group, then $G$ is totally disconnected and not polythetic and hence $G$ satisfies the assumption of our Theorem. Of course, there exist compact and totally disconnected $A$ belian groups which are neither torsion nor polythetic. For instance, the direct product $\prod_{p \in \mathcal{P}} Z(p)$ is a typical example, where $\mathcal{P}$ denotes the set of all prime numbers and $Z(p)$ is the finite cyclic group of order $p$.

(b) Lemma 1 also remains valid for any locally compact Abelian group. To see this, we have only to repeat the proof of Lemma 1 with a locally compact Abelian group $G$.

(c) It is well-known that $C(G)$ and $L^{p}(G)(1 \leqslant p<\infty)$ are Segal algebras on $G$ for any compact Abelian group $G$. Our Theorem for these Segal algebras improves and strengthens [ 8 , Theorem 4] and [6, Corollary to Theorem 6]. For a number of examples of Segal algebras other than $C(G)$ and $L^{p}(G)(1 \leqslant p<\infty)$, we refer to [12, Examples 4.12].

(d) If $G$ is an infinite compact Abelian group and if $G / C_{G}$ is polythetic, then there exist Segal algebras $S(G)$ on $G$ such that every TILF on $S(G)$ is automatically continuous. Indeed, for such $G$ 's, Johnson [2, Theorem 5.2] proved that $L^{2}(G)_{0}=$ $\triangle\left(L^{2}(G)\right)$ and hence every TILF on $L^{2}(G)$ is continuous. (For some related results, see $[1,10,11]$.) On the contrary, it is shown by Saeki $[11$, Theorem 1*] that if $G$ is a noncompact, $\sigma$-compact, locally compact Abelian group, then any Segal algebra on $G$ admits uncountably many discontinuous TILF's. Our Theorem complements this result of Saeki. The question of the existence of discontinuous TILF's on some special Segal algebras is also studied in $[3,4,5]$.

\section{REFERENCES}

[1] J. Bourgain, 'Translation-invariant forms on $L^{p}(G)(1<p<\infty)$ ', Ann. Inst. Fourier (Grenoble) 36 (1986), 97-104.

[2] B.E. Johnson, 'A proof of the translation invariant form conjecture for $L^{2}(G)$ ', Bull. Sci. Math. 107 (1983), 301-310. 
[3] C.J. Lester, 'Continuity of operators on $L^{2}(G)$ and $L^{2}(G)$ commuting with translations', J. London Math. Soc. 11 (1975), 144-146.

[4] P. Ludvik, 'Discontinuous translation-invariant linear functionals on $L^{1}(G)$ ', Studia Math. 56 (1976), 21-30.

[5] G.H. Meisters, 'Some discontinuous translation-invariant linear forms', J. Funct. Anal. 12 (1973), 199-210.

[6] G.H. Meisters, 'Some problems and results on translation-invariant linear forms', (Proc. of Conference on Radical Banach Algebras and Automatic Continuity, Long Beach, 1981): Lecture Notes in Math. 1983 975, pp. 423-444 (Springer-Verlag, Berlin, Heidelberg and New York).

[7] H. Reiter, Classical harmonic analysis and locally compact groups (Oxford Univ. Press, London, 1968).

[8] H. Reiter, ' $L$-algebras and Segal algebras': Lecture Notes in Math. 231 (Springer-Verlag, Berlin, Heidelberg and New York, 1971).

[9] W. Roelcke, L. Asam, S. Dierolf and P. Dierolf, 'Discontinuous translation invariant linear forms on $\mathcal{K}(G)$ ', Math. Ann. 239 (1979), 219-222.

[10] J. Rosenblatt, 'Translation-invariant linear forms on $L^{P}(G)$ ', Proc. Amer. Math. Soc. 94 (1985), 226-228.

[11] S. Saeki, 'Discontinuous translation invariant functionals', Trans. Amer. Math. Soc. 282 (1984), 403-414.

[12] H.C. Wang, 'Homogeneous Banach algebras': Lecture Notes in Pure and Appl. Math. (Marcel Dekker, New York, 1977).

Department of Mathematics

Kushiro Public University of Economics

4-1-1 Ashino, Kushiro

085 Japan 